(c) 2018 IEEE. Personal use of this material is permitted. Permission from IEEE must be obtained for all other uses, in any current or future media, including reprinting/republishing this material for advertising or promotional purposes, creating new collective works, for resale or redistribution to servers or lists, or reuse of any copyrighted component of this work in other works. 


\title{
0.34-THz High-Temperature Superconducting Josephson-Junction Mixer with Superior Noise and Conversion Performance
}

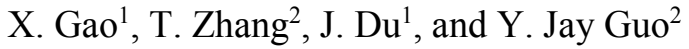 \\ ${ }^{1}$ CSIRO Manufacturing, PO Box 218, Lindfield, NSW 2070, Australia \\ ${ }^{2}$ Global Big Data Technologies Centre, University of Technology Sydney, Ultimo, NSW 2007, Australia
}

\begin{abstract}
We present, in this work, a new thin-film antenna-coupled high-temperature superconducting (HTS) Josephson-junction terahertz (THz) mixer that demonstrates superior performance at frequencies around $0.34 \mathrm{THz}$. A novel dual-meander-slot thin-film antenna is designed to significantly improve the antenna-junction impedance matching and thus more efficient coupling of the $\mathrm{THz}$ signal power. Theoretical and experimental investigations are carried out to evaluate the mixer performance. This mixer can be applied to the sensitive $\mathbf{T H z}$ wireless receivers.
\end{abstract}

\section{INTRODUCTION}

$\mathrm{T}$ HZ communication and sensing are critically important technologies for future wireless applications. Due to the limited source power and severe atmospheric attenuation at the $\mathrm{THz}$ band, supersensitive receiver frontends are desired for effective $\mathrm{THz}$ signal detection. HTS $\mathrm{THz}$ mixers are very attractive candidates due to their unique advantages like low noise, wideband response, low local oscillator (LO) pumping power, and operating at higher temperatures (thus smaller and cheaper commercial single-stage cryocooler can be used for cooling) compared to that of the low-temperature superconducting (LTS) devices. Based on the CSIRO patented YBCO step-edge junction technology [1], we have achieved major research progress in developing HTS THz narrowband [2] and broadband [3] Josephson-junction mixers. These devices are capable of operating at temperatures up to $77 \mathrm{~K}$ (liquid nitrogen temperature) and have been demonstrated excellent mixer conversion gain and noise temperature [4]. In this work, we present our new development of a novel dual-meander-slot thin-film antenna-coupled $\mathrm{HTS} \mathrm{THz}$ Josephson mixer that exhibits superior performance at the 0.34-THz band.

\section{DESIGN AND EXPERIMENT}

Quasi-optical antenna coupling offers the advantages of reliable and low-cost fabrication as well as the convenience of integration with the HTS Josephson junction. Considering the Josephson junction's normal resistance $R_{\mathrm{n}}$ is generally little (usually in the range of 1 to $10 \Omega$ ), currently reported HTS mixer antennas cannot couple $\mathrm{THz}$ radiation efficiently due to the considerable antenna-junction impedance mismatch losses. In this work, a novel $\mathrm{THz}$ lens antenna based on dual meander slots is designed, as that shown in Fig. 1, to significantly improve the impedance matching and thus $\mathrm{THz}$ power coupling. Fig. 2 shows that the antenna has a low input resistance $R_{\text {in }}$ of $\sim 13 \Omega$ at a resonant frequency of $0.34 \mathrm{THz}$. The antenna exhibits very good symmetry of the radiation patterns and achieves a coupling efficiency $\eta_{\mathrm{c}}$ as high as around $-1.7 \mathrm{~dB}$ at $0.34 \mathrm{THz}$ (see Fig. 2), the best reported result for antenna-coupled HTS Josephson-junction mixers to date.
Theoretical and experimental investigations are carried out to evaluate the mixer performance in terms of current-voltage characteristics, optimal biasing, optimal LO pumping, conversion efficiency and noise temperature, and its dependence on the Josephson junction parameters and operating temperatures. The theoretical design, simulation and experimental results will be presented at the conference.

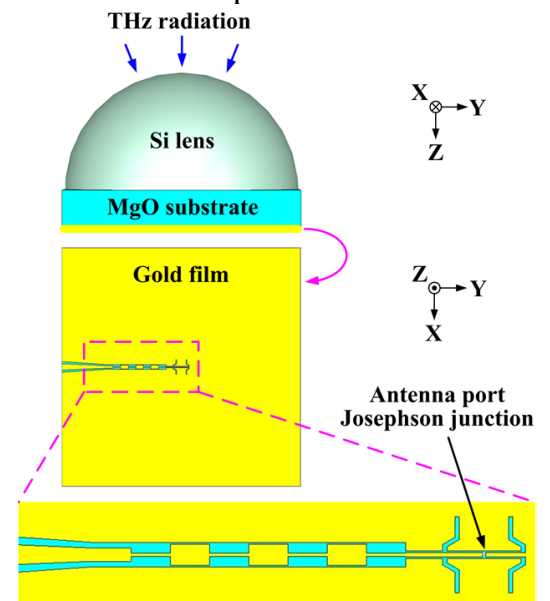

Fig. 1. Simulation model of a novel THz lens antenna based on dual meander slots. The HTS Josephson junction is integrated at the antenna's port.

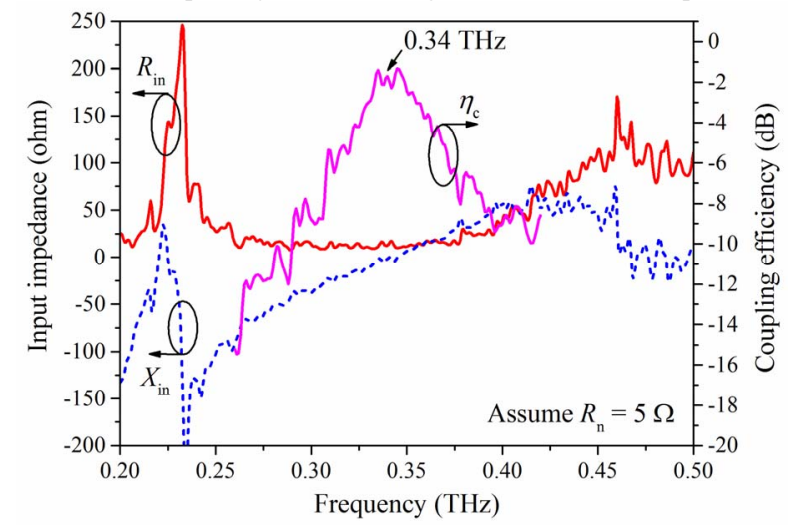

Fig. 2. Simulated input impedance and coupling efficiency of the THz antenna.

\section{REFERENCES}

[1]. C. P. Foley, E. E. Mitchell, S. K. H. Lam, B. Sankrithyan, Y. M. Wilson, D. L. Tilbrook, and S. J. Morris, "Fabrication and characterisation of YBCO single grain boundary step edge junctions," IEEE Trans. Appl. Supercond., vol. 9, no. 2, pp. 4281-4284, Jun. 1999.

[2]. J. Du, A. R. Weily, X. Gao, T. Zhang, C. P. Foley, and Y. J. Guo, "HTS step-edge Josephson junction terahertz harmonic mixer," Superconductor Science and Technology, vol. 30, no. 2, pp. 024002, Jan. 2017.

[3]. X. Gao, T. Zhang, J. Du, A. R. Weily, Y. J. Guo, and C. P. Foley, "A wideband terahertz high- $T_{\mathrm{c}}$ superconducting Josephson-junction mixer: electromagnetic design, analysis and characterization," Superconductor Science and Technology, vol. 30, no. 9, pp. 095011, Aug. 2017.

[4]. X. Gao, J. Du, T. Zhang, and Y. J. Guo, "Noise and conversion performance of a high- $T_{\mathrm{c}}$ superconducting Josephson junction mixer at 0.6 THz," Applied Physics Letters, vol. 111, no. 19, pp. 192603, Nov. 2017. 